Michel Bussi, 1998, Eléments de géographie électorale, à travers l'exemple de la France de l'Ouest

Collection Nouvelles donnes en géographie, Publications de l'Université de Rouen, Rouen, 399 p.

Dominique Badariotti

\title{
CpenEdition
}

Journals

Édition électronique

URL : http://journals.openedition.org/rge/4560

DOI : $10.4000 /$ rge. 4560

ISSN : 2108-6478

Éditeur

Association des géographes de l'Est

Édition imprimée

Date de publication : 1 janvier 1999

ISSN : 0035-3213

Référence électronique

Dominique Badariotti, « Michel Bussi, 1998, Eléments de géographie électorale, à travers l'exemple de la France de l'Ouest », Revue Géographique de l'Est [En ligne], vol. 39 / 1 | 1999, mis en ligne le 08 septembre 2013, consulté le 25 septembre 2020. URL : http://journals.openedition.org/rge/4560 ; DOI : https://doi.org/10.4000/rge.4560

Ce document a été généré automatiquement le 25 septembre 2020

Tous droits réservés 


\section{Michel Bussi, 1998, Eléments de géographie électorale, à travers l'exemple de la France de l'Ouest}

Collection Nouvelles donnes en géographie, Publications de l'Université de Rouen, Rouen, 399 p.

Dominique Badariotti

\section{RÉFÉRENCE}

Michel Bussi, 1998, Eléments de géographie électorale, à travers l'exemple de la France de l'Ouest, Collection Nouvelles donnes en géographie, Publications de l'Université de Rouen, Rouen, 399 p.

1 Les manuels de géographie électorale sont trop rares pour que l'on ne salue pas la publication d'un ouvrage venant combler ce manque.

2 Inventée par notre renommé prédécesseur, André Siegfried, la géographie électorale a connu au cours du siècle une bien étrange destinée. Lancée en France avec brio par la publication du Tableau politique de la France de l'Ouest en 1913, puis discréditée par les théories de la Geopolitik allemande, la géographie politique fut récupérée après-guerre par des historiens, des sociologues ou des anthropologues, qui l'ont peu à peu vidée de son contenu géographique. Après un long désert, marqué par une production quasimarginale, la géographie électorale renaîtra finalement au cours des années quatrevingt, dynamisée par l'utilisation de nouvelles méthodes d'analyse, quantitatives entre autres.

3 Malgré cette renaissance, l'analyse géo-électorale, pratiquée de façon expérimentale par une poignée de géographes, reste boudée, voire décriée par nos collègues politologues qui lui reprochent entre autres ses trop célèbres corrélations écologiques, et lui préfèrent toujours les méthodes de la sociologie ou de l'anthropologie. Et pourtant, l'analyse du vote peut aussi être pratiquée avec honneur et avec bonheur par 
des géographes : l'ouvrage de Michel Bussi, Éléments de géographie électorale, est là pour nous le rappeler.

4 Son ouvrage est composé de trois parties. La première brosse un tableau historique de la géographie politique et de ses outils. Elle présente l'évolution de cette discipline et de ses méthodes en remontant aux écrits ombilicaux de Seignobos ou de Tocqueville, en détaillant - à tout seigneur tout honneur - la fondation siegfriedienne de la géographie électorale, et en terminant par les contributions très récentes de Todd et de Lebras ou par celles, plus géographiques et plus techniques, de Cheylan et de Bon. Les différents courants historiques de l'analyse électorale sont décortiqués, et livrent ainsi leurs apports et leurs limites au débat géopolitique : l'époque des monographies est abordée ainsi que celle des engagements politiques ; suit une description des approches contemporaines, avec l'apparition des méthodes quantitatives et les premières expériences d'analyse spatiale géo-électorale. Les différents problèmes techniques auxquels se heurte le géopolitologue sont aussi explorés : la collecte des données et les difficultés liées aux découpages, les choix à effectuer en matière de cartographie, le délicat exercice des classifications politiques, les types d'analyses possibles, ... tous ces aspects sont tour à tour traités.

5 La seconde partie, très différente, analyse les comportements électoraux passés et actuels de la France de l'Ouest, et les confronte aux facteurs explicatifs socioéconomiques classiques, à différentes échelles. Cette partie, à la fois thématique et théorique, est une véritable démonstration de l'intérêt des analyses géographiques. Sans jamais perdre de vue le but ultime de l'opération, à savoir dresser un nouveau tableau politique de la France de l'Ouest, tout un ensemble d'analyses synchroniques et diachroniques sont effectuées, constituant ainsi une sorte d'illustration, détaillée en grandeur nature, des apports de chacune des méthodes d'analyse géo-électorale. Analyse des champs de force, analyse des mutants et de la stabilité, analyses basées sur des corrélations à différentes échelles ; tout un ensemble de techniques nous sont ici dévoilées et commentées.

Enfin la troisième partie pousse encore un peu plus loin l'essai : toujours à partir de l'exemple de l'Ouest, Bussi introduit cette fois des méthodes, basées sur des modèles anglo-saxons (Johnston ou Cox), permettant d'isoler les effets sociologiques des comportements électoraux. À partir de là, il peut se consacrer à l'analyse des autres aspects permettant de rendre compte du vote, aspects qui sont trop rarement traités dans les analyses traditionnelles du vote par les politologues. Il peut ainsi évaluer assez précisément l'influence de l'effet notabilaire, des effets éventuellement suscités par le découpage (gerrymandering, " charcutage électoral»), des effets historiques et des effets géographiques. Tous ces éléments ont bien sûr déjà été décrits dans la littérature, mais rarement leur influence aura été aussi finement évaluée, à l'aide d'outils quantitatifs. L'analyse des effets géographiques est tout à fait intéressante, notamment lorsqu'elle cherche à faire la part entre des effets de voisinage et des effets de milieux, susceptibles tous deux de jouer un rôle dans l'apparition et/ou la diffusion de votes.

On aura compris que l'intérêt de l'ouvrage de Bussi, est à la fois thématique et méthodologique. Au delà du plaidoyer pour une réhabilitation de la géographie électorale, nous retiendrons la démonstration qui indéniablement prouve que la géographie a toujours son mot à dire dans ce domaine, et que toute analyse électorale sans réflexion spatiale risque de demeurer, quelque part, incomplète. 


\section{AUTEURS}

\section{DOMINIQUE BADARIOTTI}

UFR de Géographie - Université Louis Pasteur - 3, rue de l'Argonne, 67083 Strasbourg Cedex 